\title{
Bruit et sommeil \\ Répercussions sur la santé
}

Alain Muzet

> Le sommeil est un état physiologique de récupération susceptible d'être perturbé ou raccourci sous l'effet de stimulations externes parmi lesquelles le bruit est un facteur fréquent. La perturbation du sommeil se traduit, le plus souvent, par une dégradation de la qualité de la période de veille qui lui fait suite. Les effets sur la santé d'une exposition prolongée au bruit relèvent tout autant de la qualité de vie, telle qu'appréciée subjectivement par le dormeur, que d'une atteinte vraisemblable de la fonction cardiovasculaire à travers une agression non spécifique liée au stress. <

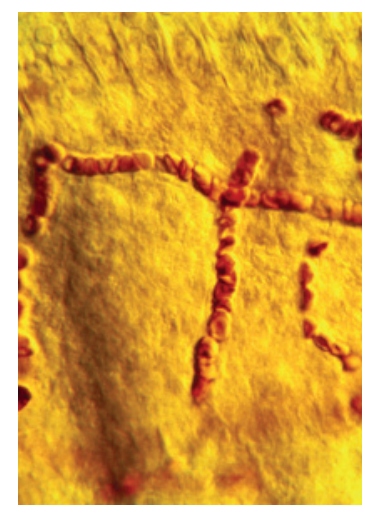

CEPA-CNRS,

21, rue Becquerel, 67087 Strasbourg Cedex, France. alain.muzet@c-strasbourg.fr

Le sommeil, état physiologique fondamental, occupe un tiers de notre vie et nous est périodiquement nécessaire afin de restaurer nos capacités physiques et mentales momentanément épuisées par nos activités de veille. Le sommeil est un état de repos relativement fragile car il peut être interrompu, volontairement ou non, par des stimulations diverses parmi lesquelles le bruit constitue une cause majeure. Cette fragilité du sommeil mérite qu'on lui accorde une attention particulière car la gêne induite, largement exprimée par les populations, peut constituer un danger par ses possibles répercussions sur la santé lors d'une exposition prolongée au bruit.

\section{Effets du bruit sur le sommeil}

\section{Exposition au bruit}

Dans notre environnement quotidien, le bruit est produit par une large variété de sources sonores parmi lesquelles les transports occupent une part prépondérante. Le bruit des avions, par exemple, est souvent incriminé de par sa spécificité et son impact important alors qu'il est très circonscrit. Le bruit des transports terrestres, quant à lui, est quasi permanent et touche des zones très étendues, notamment urbaines. Toutefois, les intensités de bruit élevées se rencontrent à proximité des sources sonores et elles s'atténuent avec la distance (Tableau I). La meilleure protection consiste donc à s'en éloigner ou à créer des barrières physiques entre les sources et les populations exposées. On peut donc dire que, contrairement à d'autres types de pollution, l'exposition au bruit est très inégalitaire car limitée à des zones bien circonscrites.

Un certain nombre d'études suggèrent que l'exposition au bruit se traduit par une demande accrue de consultations médicales. Les facteurs associés sont souvent l'appartenance à un groupe social économiquement défavorisé vraisemblable et la plus grande fragilité biologique aux agressions de cette partie de la population. Une étude réalisée dans les années 1980 par l'Institut National de Recherche sur les Transports et leur Sécurité (INRETS) montre que les ménages ayant les revenus

Article reçu le 27 février 2006, accepté le 20 août 2006.

\begin{tabular}{|c|c|c|c|c|c|c|c|c|c|c|c|}
\hline & & \multicolumn{6}{|c|}{ Seuil de l'audition } & \multirow[t]{2}{*}{ Gêne } & \multirow{2}{*}{$\begin{array}{l}\text { Fatigue } \\
\text { Cantine } \\
\text { scolaire }\end{array}$} & \multirow{2}{*}{\multicolumn{2}{|c|}{$\begin{array}{l}\text { Danger } \\
\text { Martelage }\end{array}$}} \\
\hline & $\begin{array}{c}\text { Chambre } \\
\text { sourde }\end{array}$ & & $\begin{array}{l}\text { Chambre } \\
\text { à coucher }\end{array}$ & & $\begin{array}{c}\text { Voix } \\
\text { normale }\end{array}$ & & $\begin{array}{c}\text { Salle } \\
\text { de cours }\end{array}$ & & & & \\
\hline $0 \mathrm{~dB}$ & $10 \mathrm{~dB}$ & $20 \mathrm{~dB}$ & $30 \mathrm{~dB}$ & $40 \mathrm{~dB}$ & $50 \mathrm{~dB}$ & $60 \mathrm{~dB}$ & $70 \mathrm{~dB}$ & $80 \mathrm{~dB}$ & $90 \mathrm{~dB}$ & $100 \mathrm{~dB}$ & $110 \mathrm{~dB}$ \\
\hline
\end{tabular}

Tableau I. Intensité sonore de quelques bruits de la vie courante 
les plus élevés sont quatre fois moins exposés à des intensités sonores gênantes que les ménages ayant les revenus les plus bas. De telles différences se retrouvent dans tous les tissus urbains et les personnes les plus exposées sont parfois celles qui, comme les travailleurs postés ou les travailleurs de nuit, ont besoin de se reposer au moment de la journée où les nuisances sonores sont les plus fortes.

\section{Le sommeil}

Le sommeil se présente sous la forme d'une succession d'états: les stades de sommeil, qui sont plus ou moins profonds et au cours desquels le dormeur est plus ou moins sensible aux perturbations sonores. Ces stades de sommeil sont identifiés grâce à l'enregistrement de divers paramètres physiologiques: l'activité électrique cérébrale (électroencéphalographie), les mouvements oculaires et l'électromyographie de surface auxquels sont souvent associées les variables végétatives telles que les fréquences cardiaque et respiratoire, la vasomotricité et la pression artérielle (Tableau II). La structure physiologique du sommeil est quantifiée par divers indicateurs tels que la durée de l'endormissement, la durée du sommeil, le temps passé dans chaque stade de sommeil, le nombre d'éveils et de changements de stades ainsi que les rythmes propres aux stades de sommeil. La qualité du sommeil, mais aussi la qualité de la veille qui lui fait suite, peuvent être évaluées de façon complémentaire par des questionnaires appropriés.

\section{Effets immédiats de la perturbation du sommeil}

La mise en évidence de la perturbation du sommeil implique la survenue de modifications des valeurs habituelles des indicateurs physiologiques sous l'effet du bruit et le retour à la normale de ceux-ci en l'absence de ce dernier. Aux modifications de ces indicateurs propres au sommeil, on peut également associer les modifications des variables physiologiques végétatives mesurées.

\section{Réduction du temps de sommeil}

Le temps total de sommeil peut être diminué par un allongement de l'endormissement, par des éveils nocturnes prolongés, ou encore, par un éveil prématuré non suivi d'un nouvel endormissement. II a ainsi été montré que des bruits intermittents ayant une intensité maximale de $45 \mathrm{~dB}(\mathrm{~A})$ et au-delà, peuvent augmenter la latence d'endormissement de quelques minutes à près de 20 minutes [1]. Les éveils « intra-sommeil » apparaissent dans tous les stades de sommeil avec des seuils variables mais, pour un stade donné, ces seuils d'éveil diminuent au fur et à mesure que le temps cumulé de sommeil augmente. De ce fait, lors des heures matinales, les bruits ambiants peuvent plus facilement réveiller un dormeur et l'empêcher de retrouver le sommeil. Ce réveil prématuré peut être à l'origine d'une forte réduction du temps de sommeil total.

\section{Modifications de la structure interne du sommeil}

Bon nombre des effets du bruit ne sont décelables qu'à l'aide des enregistrements physiologiques pratiqués sur le dormeur qui restent des moyens réservés aux seuls laboratoires.

- Éveils nocturnes

Comme nous l'avons vu précédemment, le bruit peut provoquer des éveils au cours du sommeil et la durée de ceux-ci peut être extrêmement variable. L'intensité du bruit nécessaire pour éveiller le dormeur dépend notamment du stade de sommeil dans lequel se trouve celui-ci. Ainsi, le seuil d'éveil (ou l'intensité sonore provoquant celui-ci) est beaucoup plus élevé dans les stades de sommeil à ondes lentes (stades 3 et 4 , souvent qualifiés de sommeil profond) que dans les stades 1 et 2 [2]. Quant au seuil d'éveil observé en sommeil paradoxal, il est extrêmement variable. Plus que dans tout autre stade de sommeil, la signification même du stimulus joue un grand rôle dans la probabilité d'observer un éveil en sommeil paradoxal [3, 4].

\begin{tabular}{|c|c|c|c|c|c|}
\hline & Stade 1 & Stade 2 & Stade 3 & Stade 4 & $\begin{array}{l}\text { Sommeil } \\
\text { paradoxal }\end{array}$ \\
\hline $\begin{array}{l}\text { Activités } \varepsilon \varepsilon G \\
\text { de fond }\end{array}$ & Ondes $\beta$ et $\theta$ & $\begin{array}{l}\text { Ondes } \theta \text { et } \delta \\
(\delta<20 \%)\end{array}$ & $\begin{array}{l}\text { Ondes } \theta \text { et } \delta \\
(20 \%<\delta<50 \%)\end{array}$ & $\begin{array}{l}\text { Ondes } \theta \text { et } \delta \\
(\delta>50 \%)\end{array}$ & Ondes $\beta$ et $\theta$ \\
\hline $\begin{array}{l}\text { Activités } \varepsilon \varepsilon G \\
\text { intermittentes }\end{array}$ & & $\begin{array}{l}\text { Fuseaux } \\
\text { Complexes K }\end{array}$ & $\begin{array}{l}\text { Fuseaux } \\
\text { Complexes K }\end{array}$ & & Ondes $\alpha$ \\
\hline $\begin{array}{l}\text { Mouvements } \\
\text { oculaires }\end{array}$ & lents & absents & absents & absents & rapides \\
\hline हMG & marqué & réduit & réduit & réduit & Très faible à absent \\
\hline $\begin{array}{l}\text { Fréquences } \\
\text { cardiaque et } \\
\text { respiratoire }\end{array}$ & Proches de l'éveil & Ralenties & $\begin{array}{l}\text { Ralenties } \\
\text { et stables }\end{array}$ & $\begin{array}{l}\text { Ralenties } \\
\text { et stables }\end{array}$ & $\begin{array}{l}\text { Augmentées } \\
\text { et variables }\end{array}$ \\
\hline
\end{tabular}

Tableau II. Critères retenus pour le diagnostic des stades de sommeil chez l'adulte. 
Ainsi, dans ce stade de sommeil particulier, le dormeur réagit plus facilement à l'appel de son nom ou de celui d'un proche qu'à l'appel du nom d'une personne qui ne lui est pas familière. De la même façon, le bruit d'une alarme peut réveiller plus facilement le dormeur qu'un bruit neutre ayant les mêmes caractéristiques physiques. Ces observations indiquent que la réaction d'un dormeur à une stimulation sonore n'est pas toujours proportionnelle à l'intensité de la stimulation et que la signification de celle-ci est parfois plus importante que ses caractéristiques physiques.

- Changements de stades de sommeil

Sous l'effet du bruit, il peut survenir des changements immédiats dans la structure interne du sommeil et notamment des changements de stades qui se font toujours dans le sens d'un allègement de celui-ci. Ces modifications ne sont pas consciemment perçues par le dormeur et il faut utiliser des enregistrements polygraphiques pour pouvoir les mettre en évidence. Ces changements de stades (souvent accompagnés de mouvements corporels) se font au détriment des stades de sommeil les plus profonds et au bénéfice des stades de sommeil les plus légers. La quantité de sommeil à ondes lentes peut être sensiblement réduite chez le jeune dormeur soumis à des bruits au cours de son sommeil [5]. Il a également été montré que la rythmicité interne du sommeil paradoxal peut être notablement perturbée lors d'une exposition nocturne au bruit $[6,7]$. Ainsi, I'instabilité du sommeil provoquée par le bruit entraîne une fragmentation de sa structure et, par là même, un amoindrissement de sa qualité, caractéristiques que l'on peut retrouver, avec une intensité variable, dans des cas d'insomnie chronique.

\section{Modifications végétatives}

Les éveils nocturnes et les modifications de la structure interne du sommeil ne sont pas les seuls effets liés à la présence des bruits. Si, dans une population générale, les éveils peuvent être obtenus pour des intensités de l'ordre de $55 \mathrm{~dB}(\mathrm{~A})$ et plus, la perturbation d'une séquence normale de sommeil peut apparaître pour des intensités comprises entre 45 et $55 \mathrm{~dB}(\mathrm{~A})$. Notons que les valeurs recommandées par l'Organisation mondiale de la santé (OMS) à l'intérieur de la chambre à coucher sont de $L_{\text {aeq,8h }}=30 \mathrm{~dB}^{1}$ et de $L_{\text {max }}=45 \mathrm{~dB}^{2}$ [8]. Cependant, cela ne signifie en aucune façon que pour des intensités maximales inférieures à ces valeurs, il n'existe plus d'effet visible du bruit. Des réponses végétatives, telles

\footnotetext{
${ }^{1} L_{\text {aeq,8h }}$ : niveau de bruit pondéré (ou équivalent) sur huit heures consécutives.

${ }^{2} L_{\text {Amax }}$ : niveau maximum (ou de crête) atteint par le bruit.
}

que des modifications du rythme cardiaque ou encore des phénomènes vasomoteurs, peuvent être observés pour des intensités de bruit bien inférieures. Ces réponses végétatives ne présenteraient en fait aucune conséquence particulière à long terme si elles étaient susceptibles de disparaître avec le temps. Or, comme nous le verrons un peu plus loin, il n'en est rien et la permanence de telles réponses végétatives sur des périodes d'exposition très longues constitue un phénomène dont on ne peut exclure, a priori, d'éventuelles conséquences cliniques à long terme sur l'organisme du dormeur [9].

\section{Effets secondaires de la perturbation du sommeil}

La mise en évidence des effets secondaires de l'exposition au bruit nocturne peut être réalisée indépendamment de l'étude des effets immédiats. Elle est, cependant, le plus souvent associée à celle-ci car elle la complète utilement. Les effets secondaires sont souvent difficiles à cerner du fait de la grande variabilité de leur latence d'apparition.

\section{Évaluations subjectives}

L'utilisation de questionnaires appropriés sert à apprécier la forme et I'humeur du dormeur au lendemain des nuits perturbées par le bruit. Ainsi, les évaluations subjectives peuvent porter sur l'estimation de la qualité du sommeil mais aussi sur la qualité de la période de veille qui lui fait suite. En effet, le coût et la complexité des enregistrements physiologiques sont tels que l'évaluation subjective reste la méthode la plus rapide et la plus accessible pour mesurer l'impact du bruit sur de très larges échantillons de populations.

Les effets de l'exposition au bruit nocturne sont le plus souvent constitués par une plainte subjective de mauvais sommeil, une mauvaise qualité de la période de veille consécutive souvent accompagnée d'une somnolence diurne et du besoin d'un repos compensateur (sieste). Ces effets secondaires peuvent exister de façon plus ou moins permanente selon la durée de l'exposition au bruit. Si le nombre de bruits est important et si les intensités de ceux-ci sont élevées, un éveil nocturne peut se transformer en éveil prolongé, voire en éveil final prématuré. C'est pourquoi les périodes initiale et finale du sommeil sont considérées comme étant les plus sensibles aux perturbations sonores $[1,10]$.

Il faut toutefois être conscient du fait que l'appréciation de la gêne ressentie peut surestimer ou sous-estimer la perturbation subie par l'organisme. En d'autres termes, les plaintes exprimées ne sont pas toujours en exacte concordance avec les mesures objectives réalisées au chevet du dormeur. Cela signifie donc que la seule prise en compte de la gêne exprimée ne permet pas d'évaluer de façon précise le coût de l'exposition au bruit. II n'est pas anodin, par exemple, de souligner que dans le cas du transport aérien, la crainte - non toujours exprimée - de la chute possible des avions peut contribuer à accentuer la plainte concernant le bruit. Cela peut d'ailleurs contribuer de façon notable à la disproportion pouvant exister entre la gêne exprimée et les intensités mesurées des dits bruits d'avion.

\section{Autres effets secondaires}

Des mesures instrumentales peuvent être utilisées pour apprécier les performances diurnes à des tâches verbales, écrites ou psycho- 
sensori-motrices plus ou moins complexes. Ainsi, il est possible de comparer les performances obtenues au lendemain de nuits perturbées par le bruit avec celles obtenues au lendemain de nuits non perturbées et de mettre en évidence une détérioration des temps de réaction consécutive à l'exposition au bruit nocturne [1]]. De façon complémentaire, il est également possible d'effectuer des dosages urinaires ou sanguins permettant de révéler l'existence de témoins biochimiques reflétant les perturbations subies par l'organisme et notamment les hormones liées au stress.

\section{Possibles répercussions du bruit sur la santé}

Les perturbations du sommeil et notamment, une forte réduction de sa durée, peuvent se traduire par une fatigue diurne excessive, souvent accompagnée par une baisse de vigilance, ou une somnolence diurne dont les effets peuvent être désastreux en termes de capacité de travail ou encore de survenue d'accidents.

\section{Perturbations du sommeil et habituation au bruit}

Un certain degré d'habituation aux conditions sonores nocturnes existe car il n'est pas rare de voir disparaître progressivement les plaintes subjectives après plusieurs jours ou semaines d'exposition au bruit. Cependant, cette habituation de l'organisme reste incomplète et les réponses végétatives observées au cours du sommeil (notamment les effets cardio-vasculaires) montrent que certaines fonctions physiologiques du dormeur restent perturbées par la répétition des perturbations sonores sur des durées d'exposition très longues [12, 13$]$. Cette non habituation physiologique au bruit est préoccupante car on ne peut négliger les effets possibles à long terme de la répétition, nuit après nuit, des perturbations sonores sur la santé des personnes exposées. Ainsi, les accélérations cardiaques, initiées de façon réflexe et observées en réponse à la plupart des bruits intenses, se répètent nuit après nuit et sont toujours mesurables après des mois et des années d'exposition au bruit alors que les personnes exposées disent souvent ne plus être gênées par le bruit! II est indéniable que ces réponses cardio-vasculaires répétées, que l'on peut qualifier d'inutiles ou de non-productives pour le dormeur, sollicitent de façon permanente le système cardiaque de celui-ci. En bordure d'une voie rapide où le trafic nocturne est de six véhicules par minute en moyenne, par exemple, on compte près de 3000 passages de véhicules pour une nuit de huit heures. En supposant que le quart seulement de ces bruits entraîne une effet mesurable, cela constitue plusieurs centaines de réactions cardio-vasculaires dont on imagine aisément qu'elles représentent un coût non négligeable pour un organisme sensé être au repos.

\section{Effets à long terme}

Bien entendu, les effets chroniques de la perturbation du sommeil par les conditions acoustiques ambiantes dépendent, en grande partie, de l'amplitude et de la fréquence de ces perturbations. II est souvent opposé qu'il n'existe pas d'évidence scientifique de conséquences cliniques indiscutables sur la santé des personnes exposées au bruit nocturne. Si l'on fait sienne la définition de la santé proposée par
I'OMS: « un état de complet bien-être physique, mental et social et non simplement une absence de maladie ou d'infirmité » [8], il n'est pas inutile de se poser la question des effets chroniques de la perturbation du sommeil par le bruit.

Il est évident qu'une perturbation du sommeil peut entraîner une réduction de la durée de celui-ci. II est cependant tout aussi clair que la durée totale de sommeil peut être modifiée dans certaines limites sans entraîner, pour autant, des modifications importantes des capacités individuelles ou du comportement. La question la plus critique, pour les praticiens de la médecine, concerne les répercussions à long terme sur la santé d'une réduction quotidienne de la durée du sommeil répétée jour après jour pendant des mois ou des années. Selon certains auteurs, le coût le plus important de la privation de sommeil pour la santé, prise ici dans son sens le plus large, est la réduction de la qualité de vie. Pour ces auteurs, la privation de sommeil entraîne une fatigue chronique excessive et de la somnolence, une réduction de la motivation de travail et une baisse des performances conduisant souvent à un sentiment de frustration et à des conflits avec les autres travailleurs. Pour des travailleurs qui ont besoin de maintenir un très haut niveau de vigilance, tels que les opérateurs de centrales nucléaires ou les contrôleurs du trafic aérien, l'anxiété liée à la privation chronique de sommeil peut être particulièrement marquée car ils savent que cette dernière affecte leurs possibilités de concentration sur leurs tâches et entraîne des baisses momentanées de l'attention. Cet état anxieux peut également se traduire par une augmentation des erreurs et peut générer des plaintes médicales associées à l'anxiété chronique.

Il a été décrit des effets généraux de l'exposition au bruit sur la santé, tant dans le cas d'une surconsommation médicamenteuse [14] que dans celui d'une augmentation des admissions dans les centres de soins psychiatriques pour les populations exposées [15]. Toutefois, de nombreux facteurs sont susceptibles d'interagir dans le cas de ces vastes études épidémiologiques et une confirmation de tels résultats est nécessaire. Des études récentes montrent l'existence d'une relation possible entre l'exposition chronique au bruit et la survenue d'hypertension, de troubles cardiaques et de problèmes médicaux sévères $[16,17]$. II n'est cependant pas inutile de relever que les services en charge de la communication des grands aéroports sont eux-mêmes susceptibles d'apporter une contribution à la connaissance des effets à long terme tels que présentés par les populations vivant à proximité de ces installations. La plupart des plaintes recensées font 
état de perturbations du sommeil, de fatigue générale et d'anxiété. Le bruit est clairement identifié comme étant un facteur de stress et le stress lui-même peut être considéré comme étant un mécanisme pouvant affecter tant la santé physique que la santé mentale des personnes exposées [18].

\section{Conclusions}

Le sommeil est donc un état au cours duquel les stimulations du monde extérieur continuent à être perçues par les organes et systèmes sensoriels de l'organisme car l'homme endormi reste susceptible de réagir aux stimulations externes. Les effets observés dépendent, bien sûr, de l'importance de l'exposition sonore nocturne et de sa répétition dans le temps. L'extraction brutale d'un univers douillet et peuplé de rêves agréables est, en soi, une agression que peu de dormeurs apprécient. Mais, c'est surtout par ses conséquences sur les activités et le bienêtre de la journée que la perturbation du sommeil est mal supportée. Cela va de la sensation de fatigue à la détérioration de certains gestes quotidiens et, notamment, de certaines activités professionnelles dont les conséquences peuvent être graves. Cela entraîne souvent la nécessité de compenser ce besoin de sommeil insatisfait par la pratique de repos compensateurs, voire d'une sieste ou, tout au moins, par une réduction prononcée du niveau d'activité. Cependant, les effets à long terme de l'exposition au bruit ne sont vraisemblablement pas différents de ceux engendrés par d'autres facteurs d'agression qu'ils soient physiques, biologiques ou psychologiques. Le bruit agit alors en combinaison avec d'autres facteurs et leurs interactions sont difficilement prévisibles. Il est possible que, chez des organismes fragilisés, le bruit soit alors le révélateur d'un état de détresse ignoré jusqu'alors. $\diamond$

\section{TIRÉS À PART}

A. Muzet

\section{SUMMARY}

The effects of noise on sleep and their possible repercussions on health

Sleep is a physiological recuperative state susceptible to be disturbed or reduced in length under external stimuli, such as noise, one of the most frequent ambient factors. Sleep disturbance is often accompanied by a reduced quality of the following waking period. The health effects of prolonged exposure to noise concern life quality, as expressed subjectively by the sleeper, as well as probable effect on cardiovascular function through a non specific aggression related to stress. $\diamond$

\section{RÉFÉRENCES}

1. Ohrström $\varepsilon$. Research on noise since 1988: present state. In : Vallet M, ed. Noise and man. Nice : INRETS, 1993 : 331-8.

2. Muzet A. Réactivité de l'homme endormi. In : Benoit 0 , Foret J, eds. Le sommeil humain. Paris : Masson, $1992:$ 77-83.

3. Oswald T, Taylor AM. Discriminative responses to stimulation during human sleep. Brain 1960 ; $83: 440-553$

4. Granda AM, Hammack JR. Operant behavior during sleep. Science $1961 ; 133: 1485-6$.

5. Carter NL. Transportation noise, sleep, and possible after-effects. Environment International $1996 ; 22: 105-16$.

6. Naitoh P, Muzet A, Lienhard JP. Effects of noise and elevated temperature on sleep cycle. In : $2^{\text {nd }}$ International Congress of Sleep research. Edimburgh, 1975.

7. Thiessen GJ. Effect of traffic noise on the cyclical nature of sleep. J Acoust Soc Am 1988 ; $84: 1741-3$.

8. WHO. Noise and health. Local authorities, health and environment. Geneva: WHO, 2000.

9. Carter NL. Cardiovascular response to environmental noise during sleep. In: $7^{\text {th }}$ International Congress on Noise as a public health problem. Sydney, Australia, 1998.

10. Fields JM. The relative effect of noise at different times of the day. NASA, Langley Research Center, 1986.

11. Wilkinson RT, Campbell KB. Effects of traffic noise on quality of sleep : assessment by દદG, subjective report, or performance the next day. J Acoust Soc Am 1984 ; 75 : 468-75.

12. Muzet $A$, Ehrhart J. Habituation of heart rate and finger pulse responses to noise during sleep. In : Tobias JV, ed. Noise as a public health problem. Rockville, Maryland: ASHA report, $n^{\circ} 10$, $1980: 401-4$

13. Vallet M, Gagneux JM, Clairet JM, et al. Heart rate reactivity to aircraft noise after a long term exposure. In : Rossi G, ed. Noise as a public health problem. Milano : Centro Ricerche e Studi Amplifon, 1983: 965-71.

14. Knipschild P, Oudshoorn N. Medical effects of aircraft noise : drug survey. Int Arch Occup Environ Health 1977 ; 40 : 197-200.

15. Tarnopolsky A, Watkins G, Hand DJ. Aircraft noise and mental health. I. Prevalence of individual symptoms. Psychol Med 1980; $10: 683-98$.

16. Maschke C. Epidemiological research on stress caused by traffic noise and its effects on high blood pressure and psychic disturbances. In : de Jong R, ed. Proceedings of ICBEN 2003. $8^{\text {th }}$ International Congress on Noise as a public health problem. Rotterdam, The Netherlands, 2003.

17. Passchier-Vermeer W. Effects of noise and health. Noise/News International 1996 : 137-50.

18. Kryter KD. The effects of noise on man. Orlando : Academic Press, 1985.

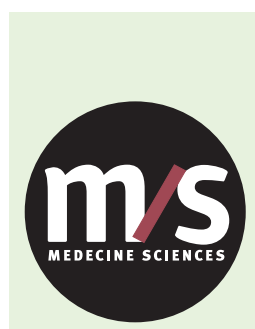

Tarifs d'abonnement M/S - 2006

> Depuis 20 ans, grâce à $\mathrm{m} / \mathrm{s}$, vous vivez en direct les progrès des sciences biologiques et médicales Abonnez-vous à Médecine/Sciences

Bulletin d'abonnement page 932 dans ce numéro de $\mathrm{m} / \mathrm{s}$

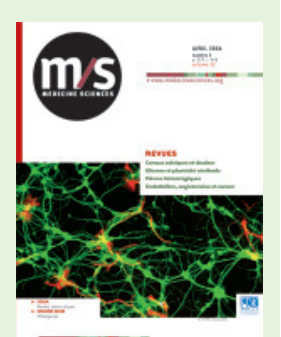

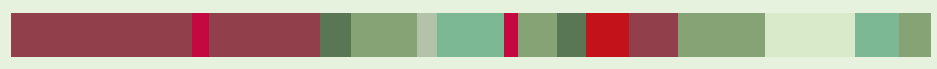


